\title{
THE NATURE OF LANGUAGE IN INTERACTION
}

\author{
ASHLEY MICKLOS
}

Applied Linguistics, University of California, Los Angeles, 3300 Rolfe Hall Box 951531 Los Angeles, CA 90095-1231, USA

\section{Abstract}

\subsection{Introduction}

This research focuses on the nature of language in interaction; specifically, how certain features of interaction (e.g. gesture, eye gaze, semiotic resources) when combined with language might facilitate communication. We extend these observations to the study of the evolution of language (as communication). Language is not used in isolation, in terms of context or form. We use language with the environment and in the environment. That is, we integrate the resources available to us when we use language, and then use those aspects jointly to accomplish tasks, goals, actions, and other interactions. Here we will study the naturalistic use of language in interaction to help parse the features that aid communicative success, expressivity, and complexity (i.e. its symbolic nature, and why that may have evolved).

\subsection{Methods}

The traditions of conversation and discourse analysis inform the current study. These methodologies consider what language, or talk, does in interaction, as well as the semiotic resources language users draw upon while attempting to accomplish actions. The data analyzed were interactions between a grandson and his grandparents performing every-day activities to accomplish a number of actions. A close examination of this data revealed how talk works with multiple resources to bring about and carry out co-operative actions- bringing together and performing operations on diverse materials (Goodwin, 2013), and how communication is facilitated by its integration of the environment, body, and other semiotic resources. In addition, a comparative analysis of cetaceans and non-human primates' interactional behavior is conducted to investigate the 
uniqueness of the organization of human action and communication as examined here. Distinctions between human and nonhuman animal co-operative behavior may shed light on the evolutionary origins of the complexity of human action and language, and how such systems help solve species-specific adaptive problems.

\subsection{Analysis}

To carry out successful communicative, members of a group often combine their talk with gestures (e.g. points, symbols, etc), meaningful eye gaze (e.g. toward a relevant object or person), and the use of objects. These resources can disambiguate the talk (and corresponding action) and help to progress the interaction. Talk itself can be quite ambiguous unless combined with these features. While the actual language might hold much expressivity, incorporating other aspects, such as gesture, can enhance it. Considering the evolution of language into a complex form of communication that is highly symbolic, we suggest the integration of other features, such as gesture, environmental resources, and the body, into our communicative interactions could allow for a more complex and symbolic communicative system to evolve as problems of communicative success could be mediated by such integration.

Humans use coordinated action and language to solve adaptive communication problems associated with cooperation and group living. Noted behavioral adaptations in other species include joint/simultaneous action and the combined use of environmental resources. But humans use these actions with a higher degree of complexity and combinatoriality, and reveal a higher degree of intersubjectivity than nonhuman animal species. Since humans share their inner worlds with one another, joint co-operational action becomes paramount to human action (Carpenter and Call, in press). We also propose the ability to combine of talk and interactional features is a learned phenomenon, wherein infants become competent participants in interaction through practice and observation. The study of language evolution might benefit from a greater consideration of the coordinated activity in which it typically manifests itself.

\section{References}

Carpenter, M., \& Call, J. (in press). How joint is the joint attention of apes and human infants? In H.S. Terrace \& J. Metcalfe (Eds.), Agency and Joint Attention. New York: Oxford University Press.

Goodwin, C. (2013). The co-operative, transformative organization of human action and knowledge. Journal of Pragmatics, 46 (1), 8-23. 